\title{
Deletions and duplications of the $22 q 11.2$ region in spermatozoa from DiGeorge/velocardiofacial fathers
}

Laia Vergés ${ }^{1 \dagger}$, Òscar Molina ${ }^{1,2+}$, Esther Geán $^{3}$, Francesca Vidal ${ }^{1}$ and Joan Blanco ${ }^{1 *}$

\begin{abstract}
Background: DiGeorge/velocardiofacial syndrome (DGSNCFS) is the most common deletion syndrome in humans. Low copy repeats flanking the 22q11.2 region confer a substrate for non-allelic homologous recombination (NAHR) events leading to rearrangements. This study sought to identify DGSNCFS fathers with increased susceptibility to deletions and duplications at the 22q11.2 region in spermatozoa and to assess the particular contribution of intra-chromatid and/or inter-chromatid NAHR. Semen samples from nine DGSNCFS fathers were analyzed by triple-color FISH using a probe combination that discriminated between normal, deleted and duplicated genotypes. Microsatellite analysis were performed in the parents and the affected children to determine the parental origin of the deleted chromosome 22.

Results: A significant increase in 22q11.2 deletions was observed in the sperm of two out of nine DGS/NCFS fathers (odds ratio 2.03-fold, $P<0.01$ ), and in both cases the deletion in the offspring was transmitted by the father. Patients with significant increases in sperm anomalies presented a disturbed deletion:duplication 1:1 ratio $(P<0.01)$.

Conclusions: Altogether, results support that intra-chromatid NAHR is the mechanism responsible for the higher rate of sperm deletions, which is directly related to the transmission of the deleted chromosome 22 to offspring. Accordingly, the screening of sperm anomalies in the $22 q 11.2$ region should be taken into account in the genetic counseling of DGSNCFS families.
\end{abstract}

Keywords: Deletions and duplications, DiGeorge/velocardiofacial syndrome, Non-allelic homologous recombination, Spermatozoa

\section{Background}

Diseases caused by chromosomal rearrangements because of architectural features of unstable regions are referred to as genomic disorders [1]. Regions involved in genomic disorders are flanked by low copy repeats (LCRs) [2] which, due to high degree of homology between their duplicated sequences ( $>95 \%)$, act as substrates for nonallelic homologous recombination (NAHR) $[3,4]$.

Depending on the LCRs orientation and the number and type of chromatids involved, different rearrangements of the intervening segments are formed (Figure 1A): complementary deletions and duplications (inter-chromatid

\footnotetext{
*Correspondence: joan.blanco@uab.cat

'Equal contributors

'Unitat de Biologia Cellular (Facultat de Biociències). Universitat Autònoma de Barcelona, 08193-Bellaterra, Cerdanyola del Vallès, Spain

Full list of author information is available at the end of the article
}

or inter-chromosomal NAHR between directly oriented LCRs), deletions and ring-acentric fragments (intrachromatid NAHR between directly oriented LCRs) or inversions (intra-chromatid NAHR between indirectly oriented LCRs) [5].

The pericentromeric area of chromosome 22 contains eight distinct highly homologous LCRs comprising roughly $11 \%$ of the 22q11.2 region. According with their position in relation to the centromere, LCR22 regions are desingated as LCR22-2, LCR22-3a, LCR22-3b, LCR22-4, LCR22-5, LCR22-6, LCR22-7 and LCR22-8 [6]. It has been reported that these LCRs are involved in recurrent reorganizations causing different genomic disorders. Among them, DiGeorge/velocardiofacial syndrome (DGS/VCFS) (OMIM $188400 /$ OMIM 192430) is the most common deletion syndrome in humans with an incidence of 1:4000 newborns 


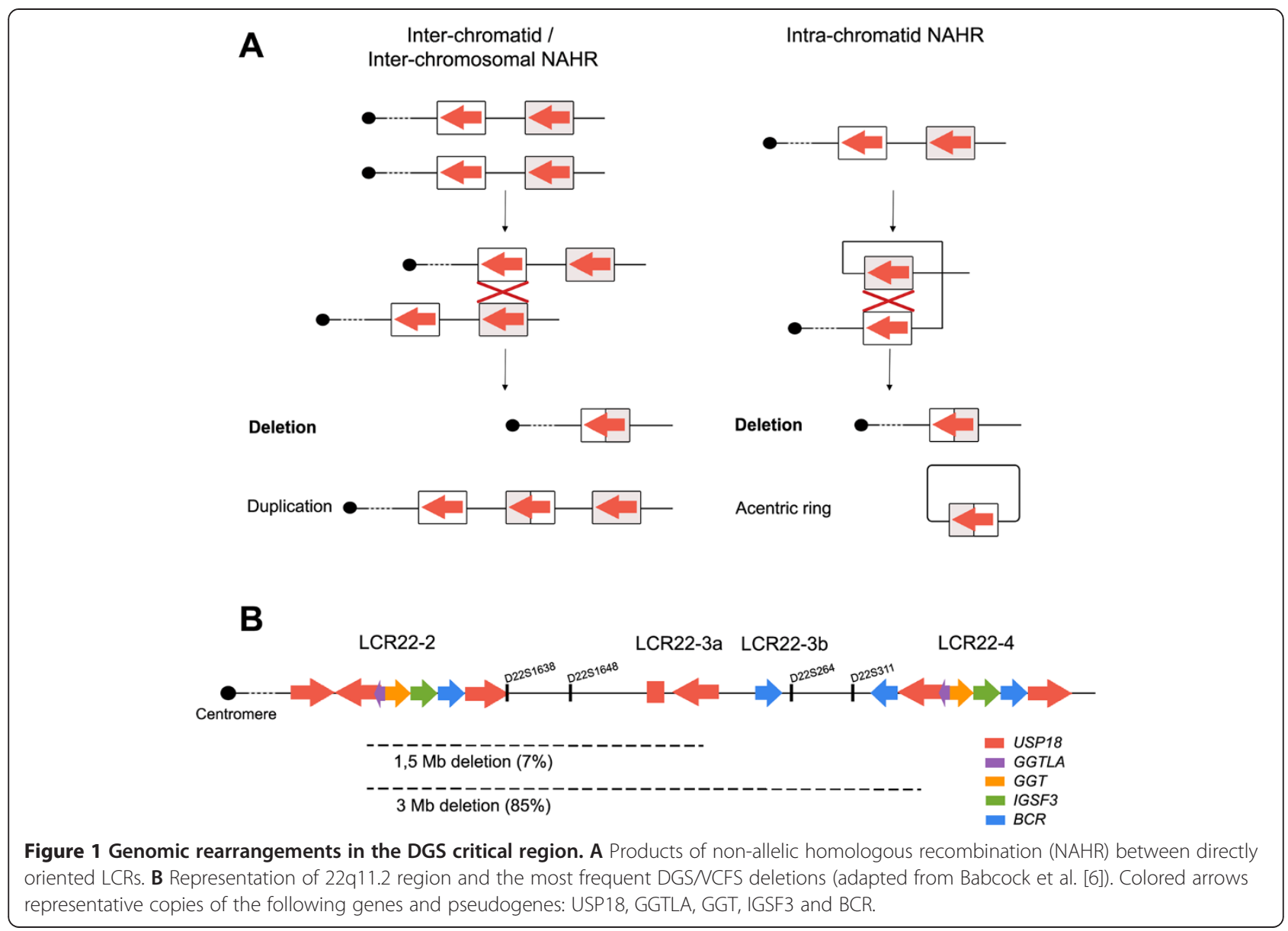

[7]. The genetic cause is the haploinsufficiency of the genes comprised within the 22q11.2 region.

More than $85 \%$ of DGS/VCFS cases are caused by NAHR events involving LCR22-2 and LCR22-4 that give rise to a $3 \mathrm{Mb}$ deletion [8]. In around 7\% of cases, there are smaller deletions of $1.5 \mathrm{Mb}$ flanked by LCR22-2 and LCR22-3a. The remaining cases are caused by unusual deletions involving distal LCR22s [9-11] (Figure 1B).

The duplication of the 22q11.2 region has been reported to cause a different phenotypic spectrum (22q11.2 duplication syndrome; OMIM 608363). It is important to mention that most of the duplications are the reciprocal products of 22q11.2 deletions originated by inter-chromatid or inter-chromosomal NAHR [12] (Figure 1A). Due to the milder phenotypes of duplications, which in some cases remain unnoticed, they have been probably underdiagnosed, thus compromising the estimation of their real incidence [13,14].

Concerning recurrence, the risk of DGS/VCFS and 22q11.2 duplication syndrome appears to be negligible, i.e. not different from the general population and has been established to be less than $0.5 \%$ [15]. The few familial cases described for DGS/VCFS have been related to a potential, unproven, parental mosaicism for a deletion in one of the progenitors [16,17]. Nevertheless, in several recurrent genomic disorders, some haplotypes have been suggested to predispose to NAHR events. In this sense, inversions of the critical regions have been reported in the fathers of children affected by deletion syndromes: Williams-Beuren [18-20], Prader-Willi [21], Angelman [22], Smith-Magenis [23] and 17q21.31 microdeletion [24], among several others [25]. In addition, copy number variations in the LCRs which flank critical regions have been described in fathers with affected offspring: SmithMagenis syndrome [26], Williams-Beuren syndrome [27] or 16p12.1 microdeletion disease [28]. Moreover trans regulator factors of meiotic recombination, such as PRDM9, have also been associated with genomic disorders. In particular, certain PRDM9 alleles have been suggested to predispose NAHR events of the regions 17p11.2 (Charcot-Marie-Tooth disease type 1A and hereditary neuropathy with liability to pressure palsies) [29], 7q11.2 (Williams-Beuren syndrome) [30] and 22q11.2 (DGS/VCFS) [31]. Finally, it has been described in the human germline an association between DNA hypomethylation of the critical regions and NAHR mediated by LCRs, suggesting the existence of an additional predisposing factor to NAHR based on methylation variations [32]. 
Thomas et al. [33] proposed a predominant role of inter-chromosomal NAHR in DGS cases, in agreement with previous observations by Edelmann et al. [34]. Similarly, previous results from our group in control donors showed no differences in the frequency of sperm $22 q 11.2$ deletions and duplications, supporting inter-chromatid/ chromosomal NAHR as the predominant mechanism for 22q11.2 rearrangements [35] (Figure 1A).

Fluorescence in situ hybridization (FISH) based methodologies in decondensed sperm nuclei allow the identification of gametes carrying deletions, duplications and inversions with a high sensitivity and specificity $[21,35,36]$. These interphase analyses, carried out in a large number of spermatozoa, offers the possibility of performing cell by cell analyses, thus assessing the incidence of rare events such as NAHR, and to establish a direct relationship between the genomic architecture and chromosomal instability during meiosis. Previous results from our group have pointed out an increased incidence of 15q11q13 deletions in spermatozoa from some Prader-Willi syndrome (PWS) fathers, suggesting a higher risk of transmission of deletions in these subjects [35].

The aim of the present study was to analyze the frequency of deletions and duplications occurring at the 22q11.2 region in spermatozoa of fathers with descendants affected by DGS/VCFS using sperm-FISH strategies. The analysis of the results will allow us to assess whether an increase in the susceptibility of generating deletions and duplications is present in these subjects and to assess the particular contribution of the intra-chromatid and/or inter-chromatid NAHR events in the generation of these anomalies.

\section{Results}

Deletion and duplication frequencies in the $22 \mathrm{q} 11.2$ region were analyzed in a total of 90,776 sperm nuclei from nine fathers of DGS/VCFS individuals (Table 1). The mean frequency of deletions $( \pm$ SEM) was $0.28 \% \pm$ 0.05 , ranging from 0.13 to $0.57 \%$ and the mean frequency of duplications $( \pm$ SEM) was $0.11 \% \pm 0.02$, ranging from 0.05 to $0.20 \%$ (Table 1 ).

No significant differences were observed in the frequency of deletions, duplications or del + dup between the population of DGS/VCFS fathers and our internal control data (Mann-Whitney test; $\mathrm{P}=0.111, \mathrm{P}=1.000$ and $\mathrm{P}=0.191$, respectively). At the individual level, we found significant increases in 22q11.2 deletions and del + dup in two out of nine DGS/VCFS fathers (Chi-square test; DG-2 and DG-5 $\mathrm{P}<0.01$ ) (Figure 2, Table 1). The risk to generate deletions of these individuals compared with the control population was estimated with an odds ratio of 2.03 (95\% CI $=1.63$ $2.54)$. On the other hand, there was no correlation between the frequencies of deletions, duplications or del + dup and the father's age (Spearman correlation; $\mathrm{P}=0.840, \mathrm{P}=0.360$ and $\mathrm{P}=0.752$, respectively).

Concerning the participation of the inter-chromatid and/or intra-chromatid NAHR in the generation of anomalies, no correlation was observed between deletion and duplication frequencies in DGS/VCFS fathers (Spearman correlation; $\mathrm{P}=0.194$ ). Furthermore, a significant increase in the mean frequency of 22q11.2 deletions was observed compared with the mean frequency of 22q11.2 duplications (Wilcoxon test; $\mathrm{P}=0.004$ ) (Figure 3). Individual comparisons showed significant increases in 22q11.2 deletions in three out of the nine cases analyzed (Chi-square test; DG-2, DG-5 and DG-6 P < 0.01) (Table 1); DG2 and

Table 1 Sperm-FISH results in the DGS fathers

\begin{tabular}{|c|c|c|c|c|c|c|c|}
\hline Case & Age & Normal & del 22q11.2 & dup $22 q 11.2$ & del + dup & Others $^{a}$ & Total \\
\hline $\mathrm{DG}-1$ & 39 & 10000 (99.19) & $24(0.24)$ & $11(0.11)$ & $35(0.35)$ & $47(0.47)$ & 10082 \\
\hline DG-2 & 48 & 10000 (98.40) & $57(0.57)^{*}$ & $20(0.20)$ & $77(0.77)^{*}$ & $86(0.86)$ & 10163 \\
\hline DG-3 & - & 9927 (99.09) & 19 (0.19) & $13(0.13)$ & $32(0.32)$ & $59(0.59)$ & 10018 \\
\hline$D G-4$ & 43 & 10000 (99.30) & $15(0.15)$ & $7(0.07)$ & $22(0.22)$ & $49(0.49)$ & 10071 \\
\hline DG-5 & 36 & 10096 (98.87) & $52(0.52)^{*}$ & $12(0.12)$ & $64(0.64)^{*}$ & $51(0.51)$ & 10211 \\
\hline DG-6 & 30 & 10000 (99.24) & $28(0.28)$ & $5(0.05)$ & $33(0.33)$ & $43(0.43)$ & 10076 \\
\hline DG-7 & 39 & 10000 (99.34) & $18(0.18)$ & $7(0.07)$ & $25(0.25)$ & $41(0.41)$ & 10066 \\
\hline DG-8 & 33 & 9948 (99.31) & $13(0.13)$ & $10(0.10)$ & $23(0.23)$ & $46(0.46)$ & 10017 \\
\hline DG-9 & 37 & 9930 (98.59) & $25(0.25)$ & $16(0.16)$ & $41(0.41)$ & $31(0.31)$ & 10072 \\
\hline$\% \pm \mathrm{SEM}$ & & $99.04 \pm 0.11$ & $0.28 \pm 0.05$ & $0.11 \pm 0.02$ & $0.39 \pm 0.06$ & $0.50 \pm 0.05$ & \\
\hline$\% \pm$ SEM $^{b}$ & & $99.08 \pm 0.09$ & $0.17 \pm 0.02$ & $0.12 \pm 0.03$ & $0.27 \pm 0.05$ & $0.64 \pm 0.05$ & \\
\hline
\end{tabular}

${ }^{\mathrm{a}}$ Disomies, diploidies and nullisomies.

${ }^{\mathrm{b} C}$ Control population data published in Molina et al., 2011 [36].

*Significant increases versus control data $(P<0.01)$.

Bold numbers indicate distortion of the 1:1 del:dup ratio $(P<0.01)$. 


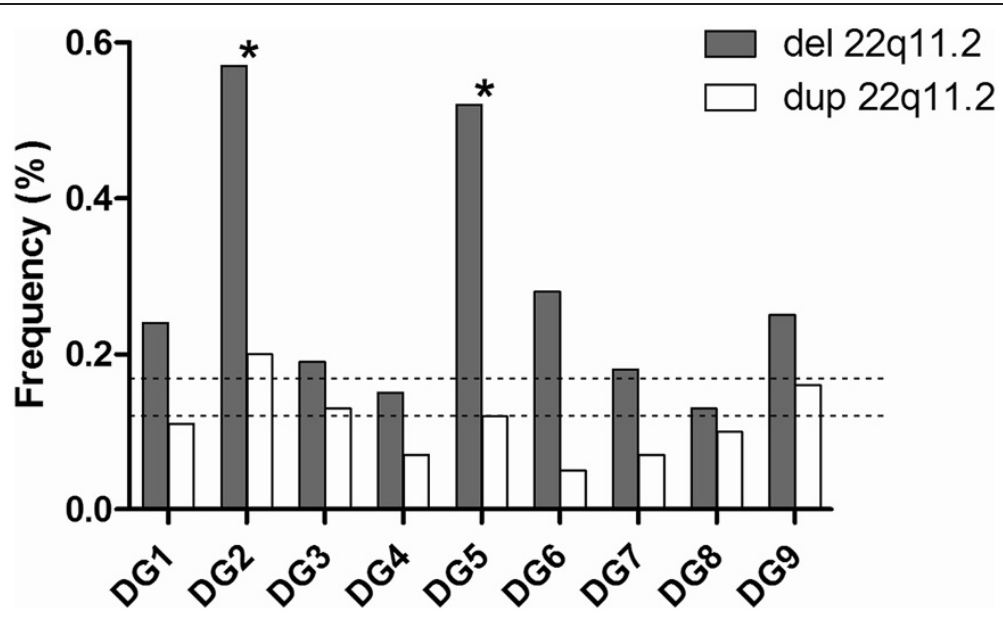

Figure 2 Frequencies of 22q11.2 deletions and duplications observed in each DGS father. Asterisks indicate cases with significant increases in deletions compared with control data (Molina et al. [36]). Dashed lines indicate deletion and duplication mean in controls (0.17 and 0.12 , respectively).

DG5 correspond to individuals that showed significant increases in 22q11.2 deletions and del + dup compared to the control data.

The parental origin of the syndromes was ascertained in eight out of the nine DGS/VCFS families. In six out of the eight families, the origin of the deletion on chromosome 22 was paternal. In the remaining two cases, the origin was maternal (Table 2). Importantly, in the fathers that showed significant increases in 22q11.2 deletions and del + dup in spermatozoa, the origin of the deletion on chromosome 22 in the affected children was paternal.

\section{Discussion}

The reliability of sperm-FISH analyses to detect deletions and duplications has already been demonstrated in previous studies $[35,36]$. The use of probes spanning the critical region, control probes for the chromosome involved and the application of strict scoring criteria allow the unequivocal identification of normal, deleted and duplicated genotypes.

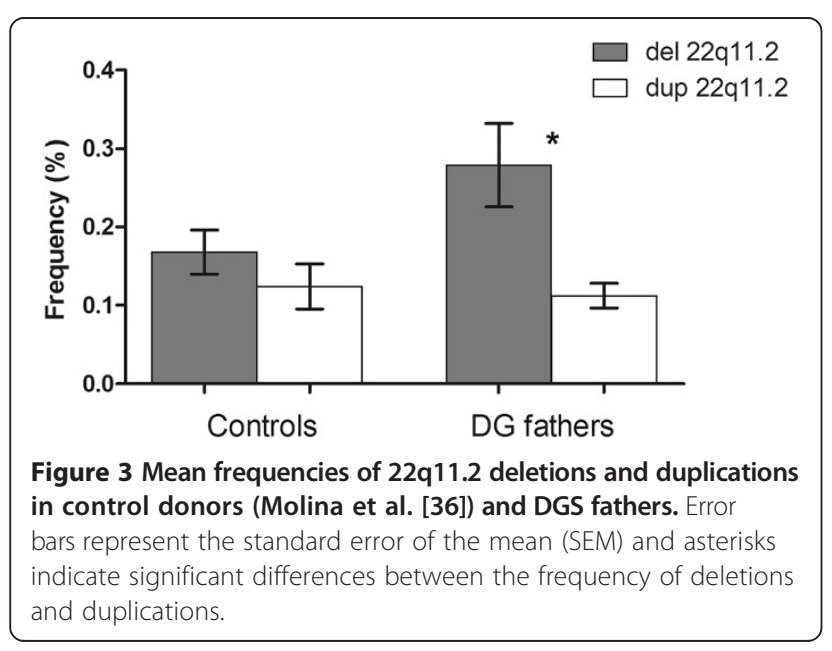

In the population of DGS/VCFS fathers, no increased susceptibility in generating deletions and duplications was observed. Nevertheless, we found significant increases in sperm 22q11.2 deletions in two fathers who were among the ones who transmitted the deletion on chromosome 22 to their offspring. Focusing on cases with a paternally inherited deletion $(n=6$; Table 2$)$, two out of the six cases $(33.3 \%)$ presented a higher risk for DGS/VCFS recurrence.

Similar situations have been observed in other studies where increases in chromosomal anomalies have been found in spermatozoa from transmitting fathers: 15q11q13 deletions in spermatozoa from Prader-Willi syndrome fathers [35], chromosome 21 disomies in spermatozoa from Down syndrome fathers [37], sex chromosomes disomies in spermatozoa from fathers with descendants affected by Turner syndrome [38] and Klinefelter syndrome [39]. Although the number of DGS/VCFS fathers studied in this work is limited and the anomaly increases observed are moderate, a comprehensive analysis of the literature suggest that increases in 22q11.2 anomalies could reach clinical relevance. Actually, the risk to generate deletions of these individuals was estimated to be twice the estimated risk in the control population (odds ratio 2.03 -fold). Accordingly, these individuals should be considered at risk of transmitting DGS/VCFS to their descendants. We do believe that, in DGS/VCFS families with a 22q11.2 deletion transmitted by the father, the screening of sperm anomalies in the 22q11.2 region using sperm FISH should be taken into account to gather information that can help in the genetic reproductive counseling of families. The detection of higher rates of deletions in father spermatozoa should be an indication for a prenatal diagnosis.

Concerning the origin of the increased amount of deletions in these two fathers, although some authors have 
Table 2 Determination of the parental origin in eight DGS families: father (F), mother (M) and affected son (S)

\begin{tabular}{|c|c|c|c|c|c|c|}
\hline CASES & D22S1638 & D22S1648 & D22S264 & D22S311 & D22S303 & PARENTAL ORIGIN \\
\hline DG1F & $114 / 116$ & $174 / 174$ & $185 / 195$ & $251 / 251$ & $220 / 222$ & \\
\hline DG1M & $116 / 116$ & $174 / 174$ & 195/195 & $250 / 256$ & $222 / 223$ & \\
\hline DG1S & 116 & 174 & 195 & 256 & $220 / 223$ & PATERNAL \\
\hline $\mathrm{DG} 2 \mathrm{~F}$ & $116 / 116$ & $170 / 172$ & $195 / 203$ & $250 / 250$ & $212 / 222$ & \\
\hline DG2M & $116 / 116$ & $172 / 172$ & $201 / 201$ & $250 / 257$ & $212 / 222$ & \\
\hline $\mathrm{DG} 2 \mathrm{~S}$ & 116 & 172 & 201 & 257 & $222 / 222$ & PATERNAL \\
\hline DG3F & $99 / 105$ & $174 / 178$ & 193/199 & $256 / 256$ & $220 / 223$ & \\
\hline DG3M & $116 / 116$ & $174 / 174$ & 193/199 & $256 / 259$ & $222 / 224$ & \\
\hline DG3S & 116 & 174 & 193 & 259 & $220 / 224$ & PATERNAL \\
\hline DG5F & $116 / 120$ & $174 / 174$ & $195 / 201$ & $250 / 256$ & $224 / 225$ & \\
\hline DG5M & $116 / 118$ & $174 / 174$ & $188 / 203$ & $256 / 256$ & $224 / 226$ & \\
\hline DG5S & - & 174 & 203 & 256 & $224 / 226$ & PATERNAL \\
\hline DG6F & $105 / 116$ & $174 / 174$ & 187/191 & $250 / 250$ & $222 / 222$ & \\
\hline DG6M & $109 / 109$ & $173 / 178$ & $185 / 193$ & $250 / 254$ & $222 / 222$ & \\
\hline DG6S & 116 & 174 & 191 & 250 & $222 / 222$ & MATERNAL \\
\hline DG7F & $109 / 120$ & $174 / 174$ & 185/199 & $250 / 250$ & $222 / 224$ & \\
\hline DG7M & $116 / 114$ & $174 / 174$ & 199/205 & $254 / 257$ & $212 / 224$ & \\
\hline DG7S & 116 & 174 & 199 & 254 & $212 / 224$ & PATERNAL \\
\hline DG8F & $114 / 114$ & $174 / 174$ & 187/199 & $252 / 256$ & $213 / 223$ & \\
\hline DG8M & $109 / 116$ & $174 / 174$ & 199/201 & $250 / 256$ & $223 / 225$ & \\
\hline DG8S & 116 & 174 & 199 & 256 & $223 / 223$ & PATERNAL \\
\hline DG9F & $116 / 116$ & $174 / 174$ & $199 / 201$ & $250 / 256$ & $212 / 222$ & \\
\hline DG9M & $111 / 122$ & $170 / 171$ & 187/191 & $250 / 254$ & $222 / 222$ & \\
\hline DG9S & 116 & 174 & 201 & 256 & $212 / 222$ & MATERNAL \\
\hline
\end{tabular}

Informative microsatellites are indicated in bold.

identified specific LCR architectures which promote genomic disorders [26-28], no predisposing haplotypes have yet been described for DGS/VCFS. Nevertheless, it is reasonable to think about the possible existence of a different LCR22s architecture in risky DGS/VCFS fathers, specifically affecting those LCRs mostly involved in the formation of deletions (LCR22-2 and LCR22-4). Baumer et al. suggested that variations in the number and position of LCRs involved in DGS/VCFS may result in higher rates of unequal crossing-over [40]. Another possible explanation comes from the influence of allelic variation at the PRDM9 locus on the 22q11.2 mutation rate. In this regard, Alemany-Schmidt et al. have recently described a higher frequency of the PRDM9 A allele in a small population of transmitting DGS/VCFS individuals suggesting a link between PRDM9 allelic variation and NAHR events [31]. However, other authors have failed to identify this association [30]. Moreover, the description of PRDM9 capacity for recognizing consensus sequences located within the LCRs, has also driven the suggestion that the activity of PRDM9 depends on the number of binding motifs and the presence of specific SNPs within the LCR sequences $[29,41]$.

In any case, further work needs to be done to establish the genetic factors that promote NAHR events in individuals with an increased risk of transmitting DGS/ VCFS. This is an important task for the future that we have already embarked on, studying the LCR22s architecture using fiber-FISH strategies [42]. We envisage that this approach would allow the establishment of a direct relationship between specific LCR haplotypes and increased rates of NAHR in spermatozoa.

Regarding the NAHR mechanisms of the formation of reorganizations in the $22 \mathrm{q} 11.2$ region, the results obtained so far by our group have shown a predominant interchromatid NAHR in the 22q11.2 region of control donors [36] in agreement with other authors which established that most cases of DGS/VCFS are due to inter-chromosomal recombination [33,34]. In the present work, although a higher frequency of 22q11.2 deletions was detected in DGS/VCFS fathers compared with the frequency of duplications, the del:dup ratio in six out of nine DGS/ 
VCFS fathers $(66.7 \%)$ was equivalent to $1: 1$, reflecting major inter-chromatid NAHR events. In the remaining three DGS/VCFS fathers, we found a significant increase in deletions versus duplications and, hence, a preponderance of intra-chromatid NAHR. Importantly, two of them were classified as individuals at risk. In fact, we have described similar results in sperm-FISH studies in Prader-Willi syndrome fathers [35], i.e. a major inter-chromatid NAHR in control donors and a major intra-chromatid NAHR that causes higher rates of 15q11q13 deletions in risky fathers.

Many changes and structural rearrangements in the chromatin structure take place during pre-meiotic, meiotic, and post-meiotic stages of spermatogenesis. These rearrangements involve the formation of double-strand breaks (DSBs) which could be a source of genomic instability along spermatogenesis [43,44]. In this way, the intra-chromatid NAHR events may occur before meiosis what would give rise to germ cell mosaicism, thus increasing the chance of transmitting a deletion $[40,45]$. Indeed, germ line mosaicism has been proposed not only as the origin of genomic disorders [16,17,46,47], but also for other syndromes such as Down syndrome [48-50], suggesting that germ line mosaicism underlies the occurrence of many genetic diseases. Intra-chromatid NAHR arising during or after meiosis would be also likely to occur and would raise the deletion rates in sperm. Therefore, although the underlying mechanism remains elusive, it seems that the major genomic instability at LCR22s in risky DGS/VCFS fathers preferentially promote intra-chromatid NAHR events that could occur along all the entire spermatogenic process. In the presence of risky haplotypes, the formation of sperm deletions by other DSB repair systems, like non-homologous end joining (NHEJ) or fork stalling and template switching (FoSTeS) should be also considered [5].

\section{Conclusions}

This study demonstrated an increased risk of transmitting DGS/VCFS in some men due to higher rates of deletions in spermatozoa. Although we are aware of the limitations of the sample size and that further work needs to be done, results deserve to be taken into consideration and the screening of 22q11.2 deletions in spermatozoa should be suggested in transmitting fathers of DGS/VCFS seeking reproductive genetic advice.

\section{Methods}

\section{Biological samples}

Semen samples were obtained from nine DGS/VCFS fathers aged between 30 and 48 years. All subjects were normozoospermic and showed normal karyotyes. Peripheral blood samples were collected from eight out of the nine DGS/VCFS families in EDTA-containing tubes (Family DG4 did not provide blood samples). Each family provided blood samples from the affected offspring and from the first-degree relatives (parents). To our knowledge, none of them had been exposed to genotoxic agents, and no history of chemotherapy, radiotherapy or chronic illness was recorded.

Parents gave their informed consent in writing to participate in the study and the protocols were approved by the Institutional Ethics Commitees of the two institutions collaborating in this study (Universitat Autònoma de Barcelona and Hospital Sant Joan de Déu, Barcelona, Spain).

\section{Fluorescence in situ hybridization on sperm}

Semen samples were processed as described previously by our group; details of sperm fixation, nuclear decondensation and FISH protocol have been described elsewhere [51]. Briefly, the sperm fraction was resuspended in hypotonic solution $(0.075 \mathrm{M} \mathrm{KCl})$ for 30 minutes at $37^{\circ} \mathrm{C}$ and fixed in methanol:acetic acid (3:1) solution. Spermatozoa were spread on a slide and kept at $-20^{\circ} \mathrm{C}$. Prior to hybridization, sperm nuclei were decondensed by slide incubation at $37^{\circ} \mathrm{C}$ in Tris buffer containing $25 \mathrm{mmol} / \mathrm{ml}$ dithiothreitol and 1\% Triton X-100 from 5 to 10 minutes.

To determine the frequency of deletions and duplications in the critical 22q11.2 region, the following probe combination was used (Figure 4): 1) locus-specific (LSI) probe for TUPLE1 localized inside the critical region at the 22q11.2 sub-band (Spectrum Orange; Abbott Molecular), 2) LSI probe for ARSA localized outside the critical region at the 22q13 sub-band (Spectrum Green; Abbott Molecular) and 3) centromeric probe recognizing the D6Z1 locus of chromosome 6 (CEP6, Spectrum Aqua; Abbot Molecular). LSI ARSA was used to discriminate between nullisomy/ disomy 22 and 22q11.2 deletion/duplication genotypes, respectively, while the CEP6 probe was used as a ploidy control.

Analyses were carried out using an Olympus BX60 epifluorescence microscope equipped with a triple-band pass filter and specific filters for Aqua, FITC and Cy3. A minimum of 10,000 sperm nuclei were analyzed for every single father in two independent FISH experiments, applying strict assessment criteria [35].

\section{Statistical analysis}

Data were analyzed using SPSS 15.0 software (SPSS Inc.; Chicago IL, USA) under the advice of the statistical service of the Universitat Autònoma de Barcelona. To avoid false positives due to the high number of spermatozoa analyzed per subject, differences were considered to be statistically significant when $\mathrm{P}<0.01$.

To assess the susceptibility in generating deletions and duplications, the mean frequency of 22q11.2 deletions, duplications and the sum of deletions and duplications (del + dup) of DGS/VCFS fathers were compared with the 


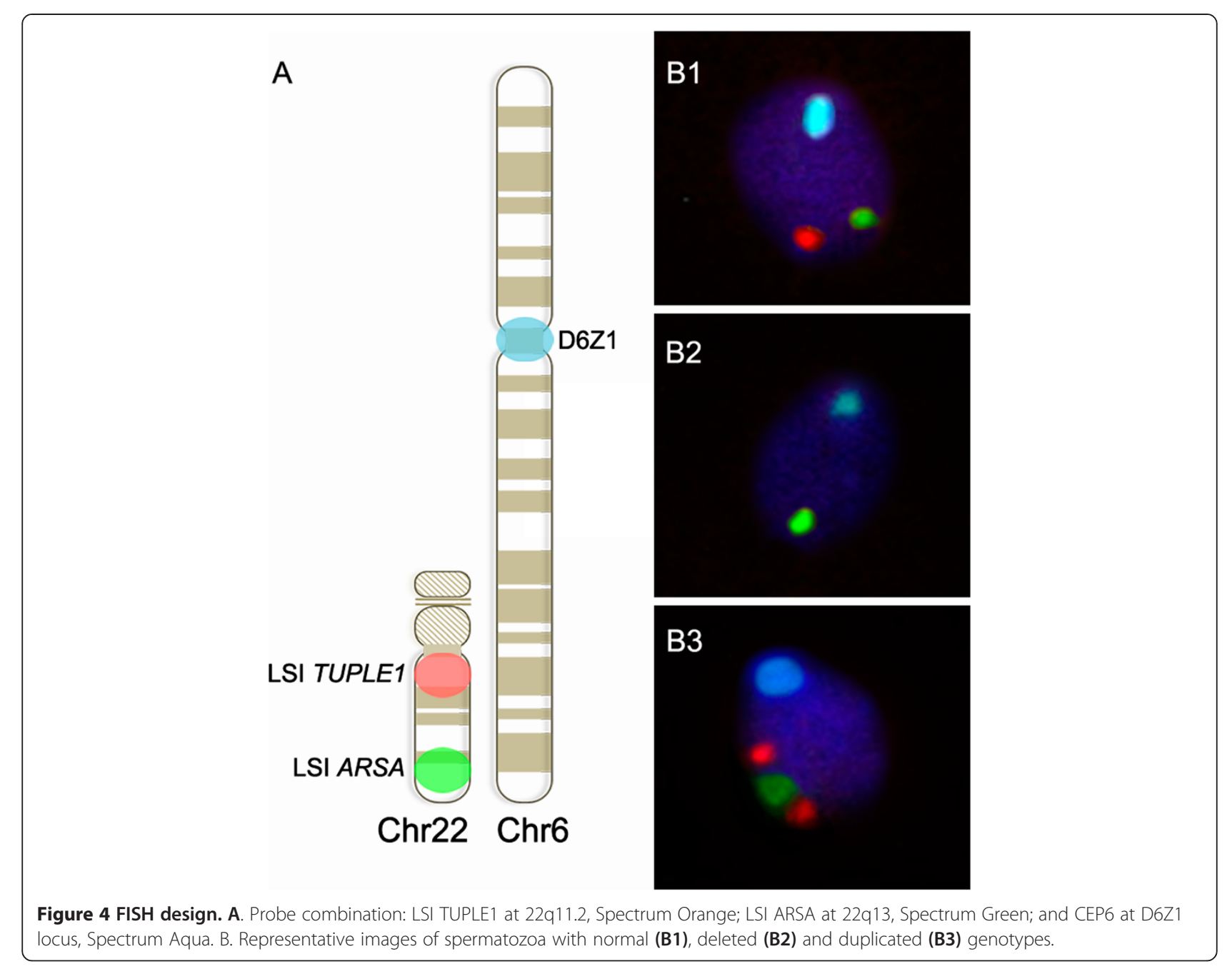

Table 3 Microsatellite markers used to determine the parental origin

\begin{tabular}{|c|c|c|c|c|c|}
\hline Microsatellite & Position & Primers & Labelling & Lenght (bp) & Annealing temperature \\
\hline \multirow[t]{2}{*}{$\mathrm{D} 22 \mathrm{~S} 1638^{\mathrm{a}}$} & $22 q 11.21$ & F: GACAACAGCAAATTGCACATT & HEX & 93 & $55^{\circ} \mathrm{C}$ \\
\hline & & R: TCACGCCACTACCCTCCAG & & & \\
\hline \multirow[t]{2}{*}{$\mathrm{D} 22 \mathrm{~S} 1648^{\mathrm{a}}$} & $22 q 11.21$ & F: CAGATGCTTCAGGAGAAGTG & HEX & 152 & $50^{\circ} \mathrm{C}$ \\
\hline & & R: AGTTGTCAGATGCCTAAGAGA & & & \\
\hline \multirow[t]{2}{*}{$\mathrm{D} 22 \mathrm{~S} 264^{\mathrm{a}}$} & $22 q 11.21$ & F: ATTAACTCATAAAGGAGCCC & HEX & 190-198 & $56^{\circ} \mathrm{C}$ \\
\hline & & R: CACCCCACCAGAGGTATTCC & & & \\
\hline \multirow[t]{2}{*}{$\mathrm{D} 22 \mathrm{~S} 311^{\mathrm{a}}$} & $22 q 11.21$ & F: GCTAGTGTGAGATAACGAAGCC & 6-FAM & 262 & $63^{\circ} \mathrm{C}$ \\
\hline & & R: TITTGTATIITAGTAGAGACGG & & & \\
\hline \multirow[t]{2}{*}{$\mathrm{D} 22 \mathrm{~S} 303^{\mathrm{b}}$} & $22 q 11.22$ & F: AGGACCTCAGACTGGTCAGTC & 6-FAM & $220-233$ & $56^{\circ} \mathrm{C}$ \\
\hline & & R: CTCCCATGAGAAGGTACACTCC & & & \\
\hline
\end{tabular}

${ }^{a}$ Microsatellites inside the deleted region.

Table shows the forward (F) and reverse (R) primers, the 5 ' labelling in the forward primers, the size of the amplified markers and their chromosomal position. 
mean frequency observed in our own control datasets [36] using a Mann-Whitney test. Next, the frequencies of deletions, duplications and del + dup of every single father were compared with the basal frequencies observed in the control population using a Chi-square test. Additionally, Spearman correlations between the frequency of deletions, duplications and del+dup and the age of DGS/VCFS fathers were performed.

To assess the participation of inter-chromatid and/or intra-chromatid NAHR in the generation of anomalies, three comparisons were performed. A Spearman correlation test of 22q11.2 deletion and duplication frequencies was performed in DGS/VCFS fathers. In order to analyze whether the mean frequency of deletions was different from that of duplications, a Wilcoxon test and Chi-square test were performed at the population and individual level, respectively.

\section{Microsatellite analysis}

To determine the parental origin of the deleted chromosome in the affected children, genomic DNA was isolated from $3 \mathrm{~mL}$ of peripheral blood samples collected in EDTAcontaining tubes using the Gentra Puregene Blood kit (QUIAGEN Inc.) following the manufacturer's instructions.

Five microsatellite markers distributed inside (D22S1638, D22S1648, D22S264, D22S311) and outside (D22S303) the deleted region were genotyped in every single DGS/VCFS family (Figure 1B; Table 3).

The microsatellite markers were amplified by PCR using dye-labeled primers (HEX or 6-FAM) and following standard procedures. PCR amplifications were performed in a reaction mixture containing 40-90 ng of genomic DNA, 25 pmol of each primer (Roche), $2.5 \mathrm{mM}$ of each dNTP (Applied Biosystems), $25 \mathrm{mM} \mathrm{MgCl}_{2}$ (Applied Biosystems), 10x PCR buffer II (Applied Biosystems) and $1 \mathrm{U}$ AmpliTaq Gold DNA polymerase (Applied Biosystems). Amplifications were performed as follows: $94^{\circ} \mathrm{C}$ for $10 \mathrm{~min} ; 35 \mathrm{cycles}$ of $94^{\circ} \mathrm{C}$ for $30 \mathrm{~s}$, an appropriate annealing temperature for $30 \mathrm{~s}$, and $72^{\circ} \mathrm{C}$ for $35 \mathrm{~s}$; and a final extension step of $72^{\circ} \mathrm{C}$ for $12 \mathrm{~min}$.

Products were analyzed by capillary electrophoresis on a genetic analyzer (ABI3130 XL, Applied Biosystems) using Peak Scanner software, version 2.0 (Applied Biosystems).

Sperm FISH and microsatellite analyses were done in a blinded manner and the results were compared only at the end of the entire experiment.

\section{Abbreviations}

FISH: Fluorescence in situ hybridization; NAHR: Nonallelic homologous recombination; LCRs: Low-copy repeats; DGSNCFS: DiGeorge/velocardiofacial syndrome.

\section{Competing interests}

The authors declare that they have no competing interests.

\section{Authors' contributions}

EG participated in providing biological samples. OM processed semen samples and carried out FISH analysis. LV and OM processed blood samples, obtained DNA and carried out microsatellite analysis. LV drafted the manuscript and participated in the stadistical analysis of the results. OM, FV and JB have been involved in revising the manuscript critically for important intellectual content. All authors read and approved the final manuscript.

\section{Acknowledgments}

This work was supported by the projects CF-180034 (Universitat Autònoma de Barcelona), Project 2014SGR-00524 (Generalitat de Catalunya, SPAIN) and SAF 2010-22241 (Ministerio de Ciencia e Inovación). Laia Vergés is recipient of a grant from the Universitat Autònoma de Barcelona (PIF/2012).

\section{Author details}

${ }^{1}$ Unitat de Biologia Cellular (Facultat de Biociències). Universitat Autònoma de Barcelona, 08193-Bellaterra, Cerdanyola del Vallès, Spain. ${ }^{2}$ Current address: Wellcome Trust Center for Cell Biology, University of Edinburgh, Edinburgh, Scotland, United Kingdom. ${ }^{3}$ Secció de Genètica Clínica. Hospital Universitari Sant Joan de Déu, 08950-Esplugues de Llobregat, Barcelona, Spain.

Received: 24 July 2014 Accepted: 4 November 2014

Published online: 25 November 2014

\section{References}

1. Lupski JR: Genomic rearrangements and sporadic disease. Nat Genet 2007, 39(7 Suppl):S43-S47.

2. Stankiewicz P, Lupski JR: Genome architecture, rearrangements and genomic disorders. Trends Genet 2002, 18:74-82.

3. Lupski JR: Genomic disorders: structural features of the genome can lead to DNA rearrangements and human disease traits. Trends Genet 1998, 14:417-422.

4. Sasaki M, Lange J, Keeney S: Genome destabilization by homologous recombination in the germ line. Nat Rev Mol Cell Biol 2010, 11:182-195.

5. Gu W, Zhang F, Lupski JR: Mechanisms for human genomic rearrangements. PathoGenetics 2008, 1:4.

6. Babcock M, Yatsenko S, Hopkins J, Brenton M, Cao Q, De Jong P, Stankiewicz P, Lupski JR, Sikela JM, Morrow BE: Hominoid lineage specific amplification of low-copy repeats on 22q11.2 (LCR22s) associated with velo-cardio-facial/digeorge syndrome. Hum Mol Genet 2007, 16:2560-2571.

7. Devriendt K, Fryns J, Mortier G, Van Thienen M, Keymolen K: The annual incidence of DiGeorge/velocardiofacial syndrome. J Med Genet 1998, 35:789-790.

8. Carlson C, Sirotkin H, Pandita R, Goldberg R, McKie J, Wadey R, Patanjali SR, Weissman SM, Anyane-Yeboa K, Warburton D, Scambler P, Shprintzen R, Kucherlapati R, Morrow BE: Molecular definition of 22q11 deletions in 151 velo-cardio-facial syndrome patients. Am J Hum Genet 1997, 61:620-629.

9. Verhagen JMA, Diderich KEM, Oudesluijs G, Mancini GMS, Eggink AJ, Verkleij-Hagoort AC, Groenenberg IAL, Willems PJ, Du Plessis FA, De Man SA, Srebniak MI, Van Opstal D, Hulsman LOM, Van Zutven LJCM, Wessels MW: Phenotypic variability of atypical $22 q 11.2$ deletions not including TBX1. Am J Med Genet A 2012, 158A:2412-2420.

10. Tan TY, Collins A, James PA, McGillivray G, Stark Z, Gordon CT, Leventer RJ, Pope K, Forbes R, Crolla JA, Ganesamoorthy D, Burgess T, Bruno DL, Slater HR, Farlie PG, Amor DJ: Phenotypic variability of distal 22q11.2 copy number abnormalities. Am J Med Genet A 2011, 155A:1623-1633.

11. Garcia-Miñaur S, Fantes J, Murray RS, Porteous MEM, Strain L, Burns JE, Stephen J, Warner JP: A novel atypical 22q11.2 distal deletion in father and son. J Med Genet 2002, 39:E62.

12. Ou Z, Stankiewicz P, Xia Z, Breman AM, Dawson B, Wiszniewska J, Szafranski P, Cooper ML, Rao M, Shao L, South ST, Coleman K, Fernhoff PM, Deray MJ, Rosengren S, Roeder ER, Enciso VB, Chinault AC, Patel A, Kang SL, Shaw CA, Lupski JR, Cheung SW: Observation and prediction of recurrent human translocations mediated by NAHR between nonhomologous chromosomes. Genome Res 2011, 2:33-46.

13. Ensenauer RE, Adeyinka A, Flynn HC, Michels W, Lindor NM, Dawson DB, Thorland EC, Lorentz CP, Goldstein JL, Mcdonald MT, Smith WE, Simon-fayard E, Alexander AA, Kulharya AS, Ketterling RP, Clark RD, Jalal SM: Microduplication 22q11.2, an Emerging Syndrome: Clinical, Cytogenetic, and Molecular Analysis of Thirteen Patients. Am J Hum Genet 2003, 73:1027-1040. 
14. Portnoï M-F: Microduplication 22q11.2: a new chromosomal syndrome. Eur J Med Genet 2009, 52:88-93.

15. Gardner RM, Sutherland GR, Shaffer LG: Chromosome Abnormalities and Genetic Counseling. 4th edition. New York: Oxford University Press; 2012.

16. Sandrin-Garcia P, Macedo C, Lr M, Es R: Recurrent 22q11.2 deletion in a sibship suggestive of parental germline mosaicism in velocardiofacial syndrome. Clin Genet 2002, 61:380-383.

17. Hatchwell E, Long F, Wilde J, Crolla J, Temple K: Molecular Confirmation of Germ Line Mosaicism for a Submicroscopic Deletion of Chromosome 22q11. Am J Med Genet 1998, 78:103-106.

18. Scherer SW, Gripp KW, Lucena J, Nicholson L, Bonnefont J, Pérez-Jurado L, Osborne LR: Observation of a parental inversion variant in a rare Williams-Beuren syndrome family with two affected children. Hum Genet 2005, 117:383-388.

19. Hobart HH, Morris CA, Mervis CB, Pani AM, Doris J, Rios CM, Kimberley KW, Gregg RG, Bray P: Inversion of the Williams syndrome region is a common polymorphism found more frequently in parents of children with Williams syndrome. Am J Med Genet C Semin Med Genet 2010, 154C:220-228

20. Osborne LR, Li M, Pober B, Chitayat D, Bodurtha J, Mandel A, Costa T, Grebe T, Cox S, Tsui L, Scherer W: A 1.5 million-base pair inversion polymorphism in families with Williams-Beuren syndrome. Nat Genet 2001, 29:321-325

21. Molina O, Anton E, Vidal F, Blanco J: High rates of de novo 15q11q13 inversions in human spermatozoa. Mol Cytogenet 2012, 5:11.

22. Gimelli G, Pujana MA, Patricelli MG, Russo S, Giardino D, Larizza L, Cheung J, Armengol L, Schinzel A, Estivill X, Zuffardi O: Genomic inversions of human chromosome 15q11-q13 in mothers of Angelman syndrome patients with class II (BP2/3) deletions. Hum Mol Genet 2003, 12:849-858.

23. Yang SP, Bidichandani SI, Figuera LE, Juyal RC, Saxon PJ, Baldini A, Patel PI: Molecular analysis of deletion (17)(p11.2p11.2) in a family segregating a $17 p$ paracentric inversion: implications for carriers of paracentric inversions. Am J Hum Genet 1997, 60:1184-1193.

24. Rao P, Li W, Vissers LELM, Veltman J, Ophoff R: Recurrent inversion events at 17q21.31 microdeletion locus are linked to the MAPT H2 haplotype. Cytogenet Genome Res 2010, 129:275-279.

25. Antonacci F, Kidd JM, Marques-Bonet T, Ventura M, Siswara P, Jiang Z, Eichler EE: Characterization of six human disease-associated inversion polymorphisms. Hum Mol Genet 2009, 18:2555-2566.

26. Carvalho CMB, Lupski JR: Copy number variation at the breakpoint region of isochromosome 17q. Genome Res 2008, 18:1724-1732.

27. Cuscó I, Corominas R, Bayés M, Flores R, Rivera-Brugués N, Campuzano V, Pérez-Jurado LA: Copy number variation at the $7 \mathrm{q} 11.23$ segmental duplications is a susceptibility factor for the Williams-Beuren syndrome deletion. Genome Res 2008, 18:683-694.

28. Antonacci F, Kidd JM, Marques-Bonet $T$, Teague B, Ventura M, Girirajan $S$, Alkan C, Campbell CD, Vives L, Malig M, Rosenfeld JA, Ballif BC, Shaffer LG, Graves TA, Wilson RK, Schwartz DC, Eichler EE: A large and complex structural polymorphism at 16p12.1 underlies microdeletion disease risk. Nat Genet 2010, 42:745-750.

29. Berg IL, Neumann R, Lam K-WG, Sarbajna S, Odenthal-Hesse L, May CA Jeffreys AJ: PRDM9 variation strongly influences recombination hot-spot activity and meiotic instability in humans. Nat Genet 2010, 42:859-863.

30. Borel C, Cheung F, Stewart H, Koolen DA, Phillips C, Thomas NS, Jacobs PA, Eliez S, Sharp AJ: Evaluation of PRDM9 variation as a risk factor for recurrent genomic disorders and chromosomal non-disjunction. Hum Genet 2012, 131:1519-1524.

31. Alemany-Schmidt A, Navarro-Palou M, Voltes-Cobo A, Rosell J, Heine-Suñer D, Picornell A, Oliver-Bonet M: Geographical genetic variability: a factor to consider when assessing clinical implications of PRDM9. Mol Genet Genomic Med 2014, 2:201-203.

32. Li J, Harris R, Cheung S, Coarfa C: Genomic hypomethylation in the human germline associates with selective structural mutability in the human genome. PLoS Genet 2012, 8:e1002692.

33. Thomas NS, Durkie M, Potts G, Sandford R, Van Zyl B, Youings S, Dennis NR, Jacobs PA: Parental and chromosomal origins of microdeletion and duplication syndromes involving $7 q 11.23,15 q 11-q 13$ and 22q11. Eur J Hum Genet 2006, 14:831-837.

34. Edelmann L, Pandita RK, Morrow BE: Low-copy repeats mediate the common 3-Mb deletion in patients with velo-cardio-facial syndrome. Am J Hum Genet 1999, 64:1076-1086.
35. Molina O, Blanco J, Vidal F: Deletions and duplications of the 15q11-q13 region in spermatozoa from Prader-Willi syndrome fathers. Mol Hum Reprod 2010, 16:320-328

36. Molina O, Anton E, Vidal F, Blanco J: Sperm rates of 7q11.23, 15q11q13 and 22q11.2 deletions and duplications: a FISH approach. Hum Genet 2011, 129:35-44.

37. Blanco J, Gabau E, Gómez D, Baena N, Guitart M, Egozcue J, Vidal F: Chromosome 21 disomy in the spermatozoa of the fathers of children with trisomy 21 , in a population with a high prevalence of Down syndrome: increased incidence in cases of paternal origin. Am J Hum Genet 1998, 63:1067-1072

38. Martínez-Pasarell O, Nogués C, Bosch M, Egozcue J, Templado C: Analysis of sex chromosome aneuploidy in sperm from fathers of Turner syndrome patients. Hum Genet 1999, 104:345-349.

39. Arnedo N, Templado C, Sánchez-Blanque Y, Rajmil O, Nogués C: Sperm aneuploidy in fathers of Klinefelter's syndrome offspring assessed by multicolour fluorescent in situ hybridization using probes for chromosomes 6, 13, 18, 21, 22, $X$ and Y. Hum Reprod 2006, 21:524-528.

40. Baumer $A$ : High level of unequal meiotic crossovers at the origin of the 22q11. 2 and 7q11.23 deletions. Hum Mol Genet 1998, 7:887-894.

41. Myers S, Freeman C, Auton A, Donnelly P, McVean G: A common sequence motif associated with recombination hot spots and genome instability in humans. Nat Genet 2008, 40:1124-1129.

42. Molina O, Blanco J, Anton E, Vidal F, Volpi EV: High-resolution fish on DNA fibers for low-copy repeats genome architecture studies. Genomics 2012 100:380-386.

43. Leduc F, Nkoma GB, Boissonneault G: Spermiogenesis and DNA repair: a possible etiology of human infertility and genetic disorders. Syst Biol Reprod Med 2008, 54:3-10.

44. Srivastava N, Raman MJ: Homologous recombination-mediated double-strand break repair in mouse testicular extracts and comparison with different germ cell stages. Cell Biochem Funct 2007, 25:75-86.

45. Dutly $F$, Schinzel A: Unequal interchromosomal rearrangements may result in elastin gene deletions causing the Williams-Beuren syndrome. Hum Mol Genet 1996, 5:1893-1898.

46. Kara-Mostefa A, Raoul O, Lyonnet S, Amiel J, Munnich A, Vekemans M, Magnier S, Ossareh B, Bonnefont J: Recurrent Williams-Beuren Syndrome in a Sibship Suggestive of Maternal Germ-Line Mosaicism. Am J Hum Genet 1999, 64:1475-1478

47. Sánchez J, Fernández R, Madruga M, Bernabeu-Wittel J, Antiñolo G, Borrego $\mathrm{S}$ : Somatic and germ-line mosaicism of deletion 15q11.2-q13 in a mother of dyzigotic twins with Angelman syndrome. Am J Med Genet A 2013, 164A:370-376.

48. Hultén MA, Patel SD, Tankimanova M, Westgren M, Papadogiannakis N, Jonsson AM, Iwarsson E: On the origin of trisomy 21 Down syndrome. Mol Cytogenet 2008, 1:21.

49. Somprasit C, Aguinaga M, Cisneros PL, Torsky S, Carson SA, Buster JE, Amato P, Lou McAdoo S, Simpson JL, Bischoff FZ: Paternal gonadal mosaicism detected in a couple with recurrent abortions undergoing PGD: FISH analysis of sperm nuclei proves valuable. Reprod Biomed Online 2004, 9:225-230.

50. Cozzi J, Conn CM, Harper J, Winston RML, Rindl M, Farndon PA, Delhanty JDA A trisomic germ cell line and precocious chromatid segregation leads to recurrent trisomy 21 conception. Hum Genet 1999, 104:23-28.

51. Sarrate Z, Anton E: Fluorescence in situ hybridization (FISH) protocol in human sperm. J Vis Exp 2009, 31:e1405.

\section{doi:10.1186/s13039-014-0086-3}

Cite this article as: Vergés et al: Deletions and duplications of the 22q11.2 region in spermatozoa from DiGeorge/velocardiofacial fathers. Molecular Cytogenetics 2014 7:86. 